\title{
Oncological and functional outcomes following robot-assisted laparoscopic radical prostatectomy at a single institution: a minimum 5-year follow-up
}

Jun-Koo Kang ${ }^{1}$, Jae-Wook Chung ${ }^{1}$, So Young Chun², Yun-Sok Ha', Seock Hwan Choi', Jun Nyung Lee ${ }^{1}$, Bum Soo Kim", Ghil Suk Yoon ${ }^{3}$, Hyun Tae Kim¹, Tae-Hwan Kim¹, Tae Gyun Kwon

${ }^{1}$ Department of Urology, Kyungpook National University School of Medicine; ${ }^{2}$ Biomedical Research Institute, Kyungpook National University Hospital; ${ }^{3}$ Department of Pathology, Kyungpook National University School of Medicine, Daegu, Korea

Background: To evaluate mid-term oncological and functional outcomes in patients with prostate cancer treated by robot-assisted laparoscopic radical prostatectomy (RALP) at our institution.

Methods: We retrospectively reviewed the medical records of 128 patients with prostate cancer who underwent RALP at our institution between February 2008 and April 2010. All patients enrolled in this study were followed up for at least 5 years. We analyzed biochemical recurrence (BCR)-free survival using a Kaplan-Meier survival curve analysis and predictive factors for BCR using multivariate Cox regression analysis. Continence recovery rate, defined as no use of urinary pads, was also evaluated.

Results: Based on the D'Amico risk classification, there were 30 low-risk patients (23.4\%), 47 intermediaterisk patients (38.8\%), and 51 high-risk patients (39.8\%), preoperatively. Based on pathological findings, $50.0 \%$ of patients $(64 / 128)$ showed non-organ confined disease ( $\geq \mathrm{T} 3 \mathrm{a})$ and $26.6 \%(34 / 128)$ had high grade disease (Gleason score $\geq 8$ ). During a median follow-up period of 71 months (range, 66-78 months), the frequency of BCR was $33.6 \%(43 / 128)$ and the median BCR-free survival was $65.9(0.4-88.0)$ months. Multivariate Cox regression analysis revealed that high grade disease (Gleason score $\geq 8$ ) was an independent predictor for $B C R$ (hazard ratio $=4.180,95 \%$ confidence interval $=1.02-17.12, p=0.047$ ). In addition, a majority of patients remained continent following the RALP procedure, without the need for additional intervention for post-prostatectomy incontinence.

Conclusion: Our study demonstrated acceptable outcomes following an initial RALP procedure, despite $50 \%$ of the patients investigated demonstrating high-risk features associated with non-organ confined disease.

Keywords: BCR; Prostate cancer; Prostatectomy; Urinary incontinence

\section{INTRODUCTION}

Prostate cancer $(\mathrm{PCa})$ is the second most commonly diagnosed cancer in the world, behind only lung cancer. It is

Received: January 25, 2018, Revised: May 30, 2018 Accepted: June 1, 2018

Corresponding Author: Tae Gyun Kwon, Department of Urology, Kyungpook National University School of Medicine, 807, Hoguk-ro, Buk-gu, Daegu 41404, Korea

Tel: +82-53-200-2166, Fax: +82-53-200-2027

E-mail: tgkwon@knu.ac.kr the most common cancer in men, accounting for $25 \%$ of all cancers affecting men. The increased incidence of PCa has resulted primarily from improved prostate specific antigen (PSA) screening, which can detect many early-stage PCa cases [1]. In Korea, PCa incidence doubled between 2007 and 2013, and the PCa mortality rate increased slightly [2]. A variety of treatment options are available for the management of localized PCa. Radical prostatectomy is a common treatment modality, which can be performed via an open, laparoscopic, or robot-assisted laparoscopic approach [3,4]. Although several reports have described short-term oncological and func-

Copyright (C) 2018 Yeungnam University College of Medicine

This is an Open Access article distributed under the terms of the Creative Commons Attribution Non-Commercial License (http://creativecommons.org/licenses/by-n/4.0/) which permits unrestricted non-commercial use, distribution, and reproduction in any medium, provided the original work is properly cited. 
tional outcomes associated with robot-assisted laparoscopic radical prostatectomy (RALP) procedures, few studies have described outcomes after a sufficiently long follow-up period. As highlighted by a previous formal systematic review, there is no solid body of evidence establishing the superiority of any one surgical approach over the others. Some studies has shown that RALP has been increasingly adopted worldwide as a surgical treatment option for localized PCa because of its minimally invasive nature and excellent oncological and functional outcomes following the procedure [5]. RALP is associated with lower perioperative morbidity and a lower rate of positive surgical margins, compared to a laparoscopic prostatectomy, although it is also associated with considerable methodological uncertainty. Some studies have reported an earlier recovery of urinary continence and erectile function in patients undergoing RALP compared to a contemporary series involving patients who underwent other prostatectomy procedures [6. However, most studies have suggested that no formal differences in the occurrence of cancer-related continence or erectile dysfunction outcomes are present [7]. We evaluated mid-term oncological and functional outcomes in patients with PCa who underwent RALP at our institution, using at least 5 years of follow-up for assessment.

\section{MATERIALS AND METHODS}

\section{Patients}

The da Vinci Surgical System (Intuitive Surgical, Sunnyvale, CA, USA) was introduced at our institution in 2008. We reviewed the records of the initial 128 patients who underwent RALP between February 2008 and April 2010. All patients were available for at least 5 years of follow-up.

Our technique for RALP is as follows: a 4-arm robot is used and a total of six ports are placed. Patients were placed on the operating table in the standard $30^{\circ}$ Trendelenburg position. Following the development of the Retzius space, we performed standard lymph node dissection (lateral limit, genitofemoral nerve; cephalad limit, bifurcation of the common iliac artery; caudal limit, endopelvic fascia; medial limit, bladder) and the preprostatic fat was removed. The bladder and prostate border were dissected first and then the bladder and prostate were divided using a Bovie knife along the bladderprostate imaginary borderline, until the prostatic urethra was exposed. The prostatic urethra was then incised and a previously placed Foley urinary catheter was observed, before continuing the division of the bladder and prostate. When the prostate was completely divided, the seminal vesicles and vas deferens were exposed and divided, and the vascular structures around them were ligated. After opening Denonvilliers' fascia, periprostatic tissue was then dissected in an antegrade fashion on each side, with scissors. Neurovascular bundles on both sides of the prostate gland were protected using an interfascial technique. With cephalad traction on the prostate, the urethra and the recto-urethralis muscle were then divided, close to the prostate. After posterior reconstruction (Rocco stitch), an urethrovesical anastomosis is performed according to the standard method. Adjuvant androgen deprivation therapy (ADT) was initiated in patients with a high probability of lymph node and distant organ metastases, with lymphovascular invasion (LVI) or margin positive 2 weeks after surgery [8].

Biochemical recurrence (BCR) was defined as an elevated PSA $\geq 0.2 \mathrm{ng} / \mathrm{mL}$ at least 6 weeks after surgery. The D’Amico classification, which evaluates the risk of recurrence following localized treatment of PCa categorizes patients into three groups based on blood PSA levels, Gleason scores, and tumor stages [4].

\section{Statistical analyses}

All data were collected based on a protocol approved by the Institutional Review Board. Analyzed variables included pathological parameters such as LVI and secondary treatments (androgen deprivation therapy or radiotherapy). Continuous variables assessed were median follow-up (in months), age of patient, body mass index (BMI), PSA, and prostate volume, measured using a transrectal ultrasound (TRUS). Categorical variables assessed were the clinical stage of disease, biopsy-related variables and the surgical Gleason score (GS), pathological stage of disease, LVI, and surgical margin status. The surgical GS was categorized as $\leq 6,7$, or $\geq 8$. BCR-free survival rates were analyzed using Kaplan-Meier survival curve and Cox regression analysis. All statistical evaluations were performed using SPSS version 18.0 for Windows (SPSS Inc., Chicago, IL, USA), and a $p$-value $<0.05$ (two-tailed) was considered statistically significant. The Chi-square test was used to determine the difference in proportions for categorical variables, while the Student t-test was used to assess continuous variables. 


\section{RESULTS}

Preoperative characteristics of patients are shown in Table 1. The mean age at RALP was $68.1 \pm 7.5$ years, and the median follow-up period was 71 months (range, 66-78 months). The mean preoperative PSA was $16.0 \pm 14.8 \mathrm{ng} / \mathrm{mL}$ and the mean prostate volume was $36.6 \pm 16.1 \mathrm{cc}$. Based on the D'Amico risk classification, patients were separated into three groups: low-,

Table 1. Preoperative characteristics of patients

\begin{tabular}{lc}
\hline Variable & \multicolumn{1}{c}{ Value } \\
\hline Median follow-up period (mon) & $\left.71(66-78)^{\mathrm{a}}\right)$ \\
Mean patient age (yr) & $68.1 \pm 7.5$ \\
Mean BMI $\left(\mathrm{kg} / \mathrm{m}^{2}\right)$ & $24.0 \pm 2.4$ \\
Mean PSA $(\mathrm{ng} / \mathrm{mL})$ & $16.0 \pm 14.8$ \\
Mean prostate volume (cc) & $36.6 \pm 16.1$ \\
Neoadjuvant hormonal therapy & $6(4.7)$ \\
D'Amico risk & \\
$\quad$ Low & $30(23.4)$ \\
Intermediate & $47(38.8)$ \\
High & $51(39.8)$ \\
\hline
\end{tabular}

Values are presented as mean \pm standard deviation or number (\%). BMI, body mass index; PSA, prostate-specific antigen.

${ }^{a)}$ Mean (minimum-maximum).

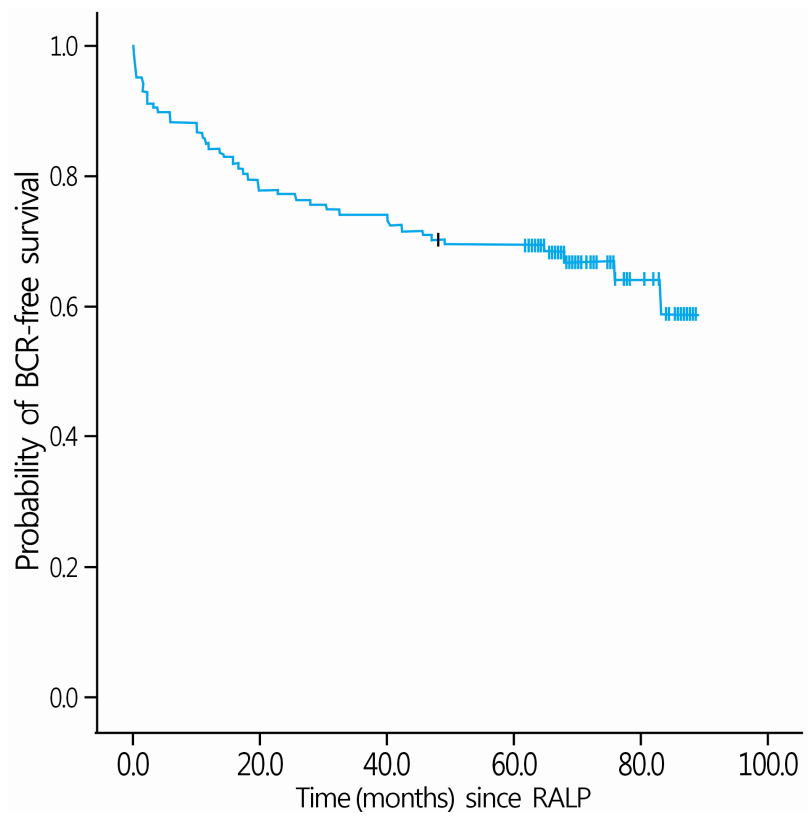

Fig. 1. Kaplan-Meier survival curve analysis of biochemical recurrence-free survival for all patients. BCR, biochemical recurrence; RALP, robot-assisted laparoscopic radical prostatectomy. intermediate-, and high-risk categories with 30 (23.4\%), 47 (38.8\%), and $51(39.8 \%)$ members, respectively.

Postoperative pathological findings and follow-up results are shown in Table 2. Organ-confined disease (T2), extraprostatic extension (T3a), and seminal vesicle invasion (T3b) were present in 64 (50.0\%), $42(32.8 \%)$, and $22(17.2 \%)$ patients, respectively. Pathologic Gleason scores of 6, 7, and 8-10 were detected in 24 (18.8\%), 70 (54.7\%), and 34 (26.6\%) patients,

Table 2. Postoperative pathological findings, follow-up results of patients

\begin{tabular}{|c|c|}
\hline Variable & Value \\
\hline \multicolumn{2}{|l|}{ Pathological $\mathrm{T}$ stage } \\
\hline $\mathrm{T} 2$ & $64(50.0)$ \\
\hline $\mathrm{T} 3 \mathrm{a}$ & $42(32.8)$ \\
\hline $\mathrm{T} 3 \mathrm{~b}$ & $22(17.2)$ \\
\hline \multicolumn{2}{|l|}{ Pathologic Gleason score } \\
\hline 6 & $24(18.7)$ \\
\hline 7 & $70(54.7)$ \\
\hline $8-10$ & $34(26.6)$ \\
\hline \multicolumn{2}{|l|}{ Pathological nodal stage } \\
\hline N0 & $125(97.7)$ \\
\hline $\mathrm{N} 1$ & $3(2.3)$ \\
\hline \multicolumn{2}{|l|}{ Lymphovascular invasion } \\
\hline No & $112(87.5)$ \\
\hline Yes & $16(12.5)$ \\
\hline \multicolumn{2}{|l|}{ Surgical margin status } \\
\hline Negative & $63(49.2)$ \\
\hline Positive & $65(50.8)$ \\
\hline $\mathrm{T} 2$ & 20/64 (31.3) \\
\hline T3a & $30 / 42(71.4)$ \\
\hline $\mathrm{T} 3 \mathrm{~b}$ & $15 / 22(68.2)$ \\
\hline BCR & $43(33.6)$ \\
\hline Median BCR-free survival, months & $65.9(0.4-88.0)$ \\
\hline Distant metastasis & $4(3.1)$ \\
\hline \multicolumn{2}{|l|}{ Adjuvant Androgen deprivation therapy } \\
\hline No & $70(54.7)$ \\
\hline Yes & $58(45.3)$ \\
\hline Duration, months & $13.73(3-55)$ \\
\hline Salvage radiotherapy & $4(3.1)$ \\
\hline Chemotherapy & $4(3.1)$ \\
\hline \multicolumn{2}{|l|}{ Mortality } \\
\hline Overall death & $3(2.3)$ \\
\hline Death from $\mathrm{PCa}$ & $2(1.6)$ \\
\hline
\end{tabular}


Jun-Koo Kang et al.

Table 3. Factors associated with biochemical recurrence (BCR)

\begin{tabular}{lccc}
\hline Variable & $\begin{array}{c}\text { BCR }(-) \\
(\mathrm{n}=85,66.4 \%)\end{array}$ & $\begin{array}{c}\text { BCR }(+) \\
(\mathrm{n}=43,33.6 \%)\end{array}$ & $p$-value \\
\hline Age $(\mathrm{yr})$ & $69.0 \pm 8.0$ & $67.4 \pm 6.6$ & 0.275 \\
BMI $\left(\mathrm{kg} / \mathrm{m}^{2}\right)$ & $24.1 \pm 2.5$ & $24.0 \pm 2.1$ & 0.589 \\
D'Amico risk & & & 0.003 \\
$\quad$ Low & $22(25.9)$ & $8(18.6)$ & \\
Intermediate & $38(44.7)$ & $9(20.9)$ & \\
$\quad$ High & $25(29.4)$ & $26(60.5)$ & \\
Preoperative PSA (ng/mL) & $13.4 \pm 9.8$ & $20.6 \pm 20.1$ & 0.013 \\
Prostate volume on TRUS (cc) & $37.3 \pm 17.5$ & $35.1 \pm 12.7$ & 0.522 \\
Pathological stage & & & 0.001 \\
$\quad$ T2 & $47(55.3)$ & $17(39.5)$ & \\
T3a & $31(36.5)$ & $11(25.6)$ & \\
T3b & $7(8.2)$ & $15(34.9)$ & \\
Pathologic Gleason score & & & \\
6 & $21(24.7)$ & $3(7.0)$ & \\
7 & $48(56.5)$ & $22(51.2)$ & \\
$\quad 8-10$ & $16(18.8)$ & $18(41.9)$ & \\
Lymphovascular invasion $(+)$ & $7(8.2)$ & $9(20.9)$ & 0.041 \\
Surgical margin status $(+)$ & $40(47.1)$ & $25(58.1)$ & 0.159 \\
\hline
\end{tabular}

Values are presented as mean \pm standard deviation or number (\%).

BMI, body mass index; PSA, prostate-specific antigen; TRUS, transrectal ultrasound.

respectively. Pathological stage N1 disease was detected in $3(2.3 \%)$ patients. Positive surgical margin was observed in 65 patients $(50.8 \%)$, and LVI was detected in $16(12.5 \%)$ patients. The overall BCR rate was 33.6\% (43/128 patients), and median BCR-free survival was 65.9 months (range, 0.4-88.0 months) (Fig. 1, Table 2). Adjuvant ADT following RALP was administered to 58 patients (45.3\%). Among all patients, four patients underwent salvage radiotherapy and four patients received chemotherapy due to the development of castration resistant prostate cancer. We found that three (2.3\%) patients died during the follow-up period; two of these deaths were determined to be disease-related.

Univariate analysis demonstrated that the high-risk category based on the D'Amico classification $(p=0.003)$, elevated preoperative PSA ( $p=0.013)$, pathologic T stage $(p=0.001)$, LVI $(p=0.041)$, and GS $\geq 8(p=0.005)$ were significantly associated with an increased risk of developing BCR (Table 3). Age, $\mathrm{BMI}$, and prostate volume were not associated with BCR. Multivariate Cox regression analysis showed that a GS $\geq 8$ was the only independent predictor of BCR (hazard ration $[\mathrm{HR}]=$ 4.180, 95\% confidence interval $[\mathrm{CI}]=1.02-17.12, p=0.047$ ) (Table 4).
Table 4. Multivariate Cox regression analysis of variables for predicting biochemical recurrence (BCR)

\begin{tabular}{|c|c|c|c|c|}
\hline Variable & \multirow[t]{2}{*}{$\mathrm{HR}$} & $\frac{95 \%}{\text { Lower }}$ & $95 \% \mathrm{CI}$ & $p$ value \\
\hline$<\mathrm{BCR}>$ & & & & \\
\hline Preoperative PSA (ng/mL) & 1.030 & 0.982 & 1.079 & 0.223 \\
\hline \multicolumn{5}{|l|}{ D'Amico risk classification } \\
\hline Low & 1 & & & \\
\hline Intermediate & 0.495 & 0.184 & 1.331 & 0.164 \\
\hline High & 1.322 & 0.544 & 3.215 & 0.538 \\
\hline \multicolumn{5}{|l|}{ Pathological stage } \\
\hline $\mathrm{T} 2$ & 1 & & & \\
\hline T3a & 0.558 & 0.244 & 1.277 & 0.167 \\
\hline $\mathrm{T} 3 \mathrm{~b}$ & 1.998 & 0.925 & 4.317 & 0.078 \\
\hline \multicolumn{5}{|l|}{ Pathologic Gleason score } \\
\hline 6 & 1 & & & \\
\hline 7 & 3.163 & 0.875 & 11.433 & 0.079 \\
\hline $8-10$ & 4.180 & 1.020 & 17.121 & 0.047 \\
\hline Lymphovascular invasion (no vs. yes) & 1.555 & 0.710 & 3.404 & 0.270 \\
\hline
\end{tabular}

BCR-free survival periods based on the preoperative D'Amico risk classification were 76.5 months, 67.5 months, and 50.7 
months, for the low-, intermediate-, and high-risk groups, respectively ( $p=0.001$, Fig. 2A). Stratifying patients based on the pathological stage of disease showed that BCR-free survival periods were 70.8 months for organ-confined disease (T2), 66.3 months for T3a stage disease, and 38.7 months for T3b $(p<0.001$, Fig. 2B). BCR-free survival periods, stratified by the Gleason score showed 79.8 months, 66.5 months, and 50.0 months for a GS $\leq 6,7$, or $\geq 8$, respectively ( $p=0.001$, Fig. 2C). The presence of LVI also showed a statistically signi- ficant difference in terms of BCR-free survival rates (LVI (-), 66.8 months; LVI (+) 46.7 months; $p=0.036$ ) (Fig. 2D).

At the 1-year postoperative follow-up, we found that 33/ $128(25.8 \%)$ patients experienced incontinence. However, 30 of 33 patients with urinary incontinence required only safety pads while the remaining three required additional intervention such as artificial urethral sphincter (AUR) implantation, for significant post-prostatectomy incontinence. Based on univariate analysis, a statistically significant difference was
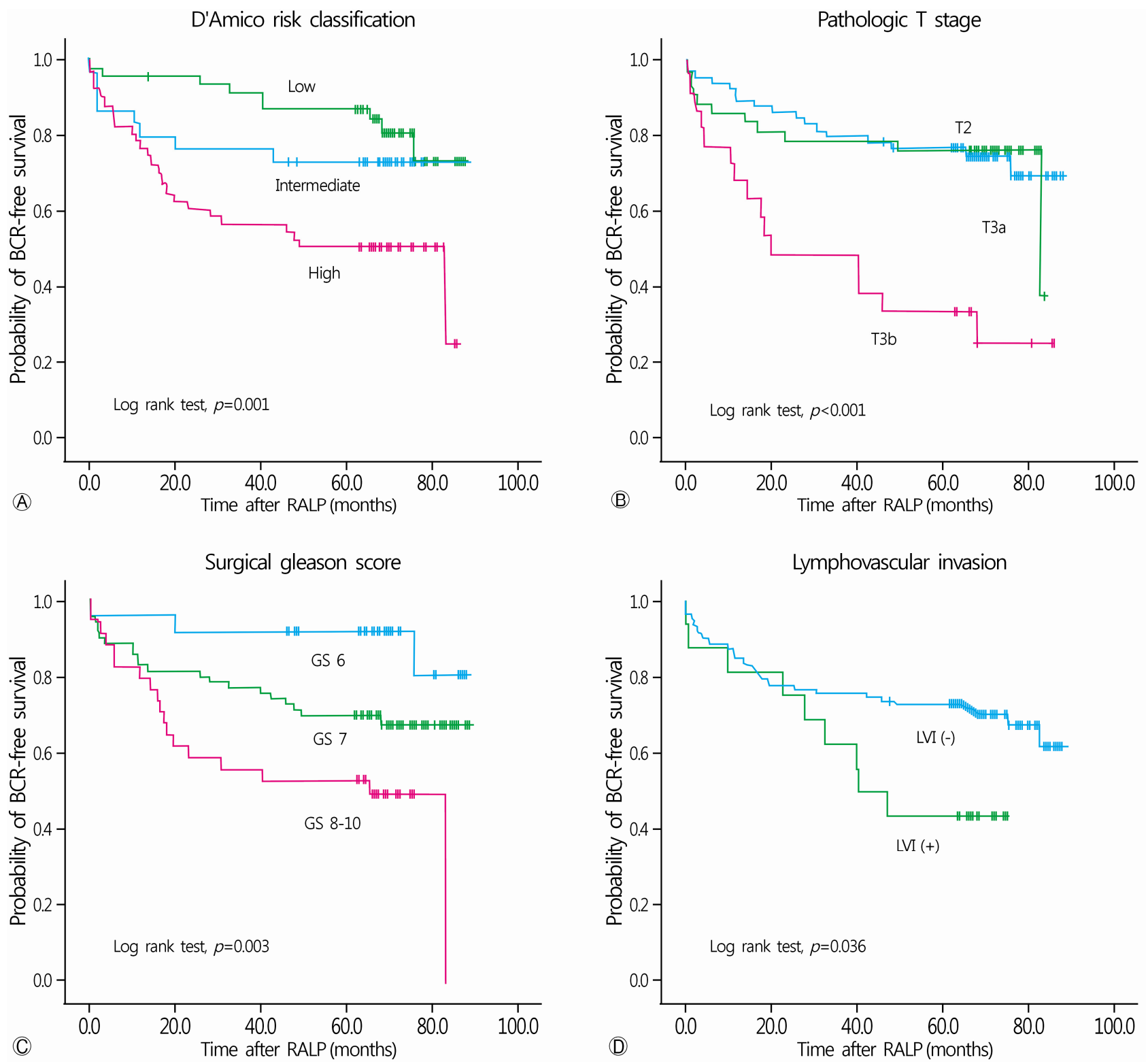

Fig. 2. Kaplan-Meier survival curve analysis of biochemical recurrence-free survival comparing the three D'Amico risk groups (A), patients based on pathological staging (B), Gleason score (C), and Lymphovascular invasion (D), using the log-rank test. RALP, robotassisted laparoscopic radical prostatectomy. 
Table 5. Univariate and multivariate analysis for predicting incontinence

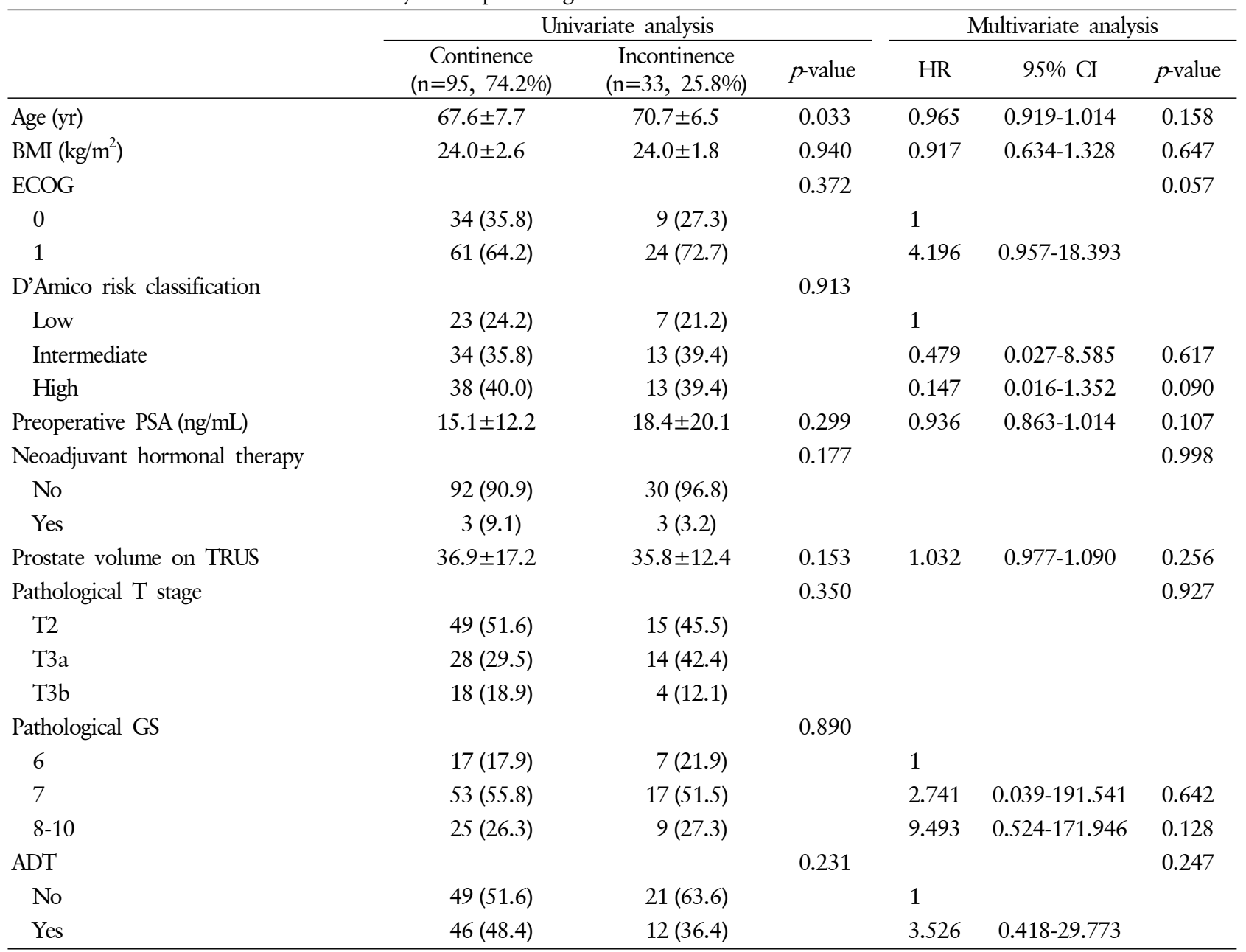

Values are presented as mean \pm standard deviation or number (\%).

BMI, body mass index; ECOG, ECOG performance status; PSA, prostate-specific antigen; TRUS, transrectal ultrasound; GS, Gleason score; ADT, androgen deprivation therapy.

noted in terms of age between the continence and incontinence groups, however, no such statistically significant differences were observed using multivariate analysis (Table 5).

\section{DISCUSSION}

In our study, we analyzed mid-term oncological and functional outcomes in patients with PCa who underwent RALP at our institution. Fifty-one (39.8\%) patients were classified as belonging to the high-risk group on preoperative D'Amico risk stratification. Postoperative pathologic results showed 34 (26.6\%) patients with GS $\geq 8$ and 64 (50\%) of patients with $\mathrm{T} 3$, suggesting a relatively high ratio of high-risk patients. BCR was found in 43 (33.6\%) patients and the median BCR- free survival period was $65.9(0.4-88.0)$ months. The presence of high grade disease (Gleason score $\geq 8$ ) was an independent factor affecting BCR development. At 1-year follow-up, urinary incontinence occurred in $33(25.8 \%)$ patients, but only three patients required AUS. There were no factors influencing post-operative urinary incontinence.

In several studies of 400 to 1,384 men treated by RALP, the median time to BCR was 2.3-2.9 years. BCR-free survival rates were $74.0-87.1 \%$ and $81.0-84.5 \%$, at 5 and 7 years [9-11]. According to BCR-free survival rate stratified by the D'Amico risk group in several studies, BCR-free survival rates were $96.8 \%, 95.1 \%$ and $92-92.6 \%$ in low-risk patients; $86.7 \%, 80.2 \%$ and $69.8-75 \%$ in intermediate-risk patients; and $78.2 \%, 72 \%$, and $64-67.5 \%$ in high-risk patients at 3, 
5, and 7 years after RALP, respectively [9,11,12]. In an Asian study, Abdel Raheem et al. [13] investigated 800 patients who underwent RALP with a median 64 months of follow-up. They revealed that BCR rates at 5-year follow-up were 54\%, $45 \%$ and $58.1 \%$ for a high-risk D'Amico, GS $\geq 8$, and clinical disease stage $\geq c T 2 c$, respectively. In our study, BCR-free survival rates were $71,69.4$, and $67.0 \%$ at $40-, 60$-, and 80months follow-up, respectively. Based on assessment of patients using the D'Amico risk classification, the 5-year BCRfree survival rate was $87.0 \%$ for the low-risk, $73.3 \%$ for the intermediate-risk, and 51.0\% for the high-risk groups. According to pathologic T stage and GS, 5-year BCR-free survival rate were $76.2 \%$ for $\mathrm{pT} 2$ stage cancers, $76.5 \%$ for pT3a stage cancers, and $34.1 \%$ for T3b stage cancers and $91.7 \%$ for GS6, 67.5\% for GS7, and 49.2\% for GS 8-10. Overall BCR rate in our study was higher than that in other studies. We suggest that the higher BCR rate in this study was due to the initial experience performed with the first introduced robot system, and a relatively high proportion of high-risk group patients.

There are several views on the predictive factors of BCR when reviewing several studies. Rajan et al. [10] showed that preoperative PSA, GS, cT stage, $\mathrm{pT}$ stage, and $>3 \mathrm{~mm}$ multifocal PSMs were predictive factors of BCR. Lee et al. [12] showed that $\mathrm{cT}$ stage, $\mathrm{pT}$ stage, and pathological GS were independent predictive factors of BCR. Diaz et al. [14] suggested that D'Amico risk groups, pathologic GS, pathologic stage, and surgical margin status were predictive factors. Sooriakumaran et al. [11] revealed that preoperative PSA, pathologic GS, pathologic stage, surgical margin status and reduced surgeon volume were predictive factors. In this study, we observed that the high-grade disease ( $G S \geq 8$ ) was a predictive factor affecting BCR.

With respect to functional outcomes, a recent study showed that 366 men (21.3\%) were incontinent 12 months after RALP [7]. Another study observed that 114 (63.3\%) patients had post-prostatectomy incontinence (PPI) 1 month after RALP, and PPI persisted in 19 (16.0\%) patients at 24 months [15]. The PPI rate in our study seems to be similar with other studies, despite the result based on early experiences of RALP. The factors affecting incontinence after surgery remain controversial. Some studies showed that the length of the membranous urethra and anatomical grades of nerve sparing can be risk factors associated with incontinence after RALP
[16,17]. Another study [15] revealed that older age and longer operative time were highly relevant to short- and longterm PPI occurrence after RALP. In this retrospective study, we did not identify any significant factor for PPI.

This study has several limitations. First, our study was a retrospective and not a randomized, case-controlled study. Thus, detailed analysis was not performed for patient characteristics such as preoperative voiding patterns. Second, our data were based on results of surgery performed when the surgeon was uncomfortable with robot system, so the oncological outcomes of surgery might not be completely valid as they could represent results obtained during the surgeons' learning curves. Third, our data did not provide information regarding patients' potency and whether or not a nerve-sparing procedure had been performed. Therefore, we could not effectively analyze and comment upon the restoration of postoperative erectile function and effect of nerve-sparing procedures on postoperative continence or BCR-free survival rates. Fourth, we did not use a questionnaire to conduct patient interviews. Therefore, we subjectively divided patients into continence and incontinence groups based on chart reviews.

In conclusion, our study demonstrated acceptable outcomes following an initial RALP procedure, although $50 \%$ of the patients investigated demonstrated high-risk features associated with non-organ confined disease.

\section{ACKNOWLEDGEMENTS}

This research was supported by the Basic Science Research Program through the National Research Foundation of Korea (NRF), which is funded by the Ministry of Science, ICT \& Future Planning (2014M3A9D3034164) (2014R1A1A3049460). Additional funding was provided by the Korean government (MSIP) (2015R1C1A1A01053509) (2016R1C1B1011180) and the Ministry of Education (2015R1D1A3A03020378).

\section{CONFLICT OF INTEREST}

No potential conflict of interest relevant to this article were reported.

\section{ORCID}

Jun-Koo Kang, https://orcid.org/0000-0002-8857-3406 
Tae Gyun Kwon, https://orcid.org/0000-0002-4390-0952

\section{REFERENCES}

1. Siegel RL, Miller KD, Jemal A. Cancer statistics, 2015. CA Cancer J Clin 2015;65:5-29.

2. Han HH, Park JW, Na JC, Chung BH, Kim CS, Ko WJ. Epidemiology of prostate cancer in South Korea. Prostate Int 2015;3:99-102.

3. Chopra S, Srivastava A, Tewari A. Robotic radical prostatectomy: the new gold standard. Arab J Urol 2012;10:23-31.

4. D'Amico AV, Moul J, Carroll PR, Sun L, Lubeck D, Chen MH. Cancer-specific mortality after surgery or radiation for patients with clinically localized prostate cancer managed during the prostate-specific antigen era. J Clin Oncol 2003;21: 2163-72.

5. Luciani LG, Mattevi D, Mantovani W, Cai T, Chiodini S, Vattovani V, et al. Retropubic, laparoscopic, and robot-assisted radical prostatectomy: a comparative analysis of the surgical outcomes in a single regional center. Curr Urol 2017;11:36-41.

6. Nossiter J, Sujenthiran A, Charman SC, Cathcart PJ, Aggarwal A, Payne $\mathrm{H}$, et al. Robot-assisted radical prostatectomy vs laparoscopic and open retropubic radical prostatectomy: functional outcomes 18 months after diagnosis from a national cohort study in England. Br J Cancer 2018;118:489-94.

7. Haglind E, Carlsson S, Stranne J, Wallerstedt A, Wilderäng $\mathrm{U}$, Thorsteinsdottir $\mathrm{T}$, et al. Urinary incontinence and erectile dysfunction after robotic versus open radical prostatectomy: a prospective, controlled, nonrandomised trial. Eur Urol 2015; 68:216-25.

8. Kang YJ, Kim HS, Jang WS, Kwon JK, Yoon CY, Lee JY, et al. Impact of lymphovascular invasion on lymph node metastasis for patients undergoing radical prostatectomy with negative resection margin. BMC Cancer 2017;17:321.

9. Menon M, Bhandari M, Gupta N, Lane Z, Peabody JO, Rogers
CG, et al. Biochemical recurrence following robot-assisted radical prostatectomy: analysis of 1384 patients with a median 5-year follow-up. Eur Urol 2010;58:838-46.

10. Rajan P, Hagman A, Sooriakumaran P, Nyberg T, Wallerstedt A, Adding C, et al. Oncologic outcomes after robot-assisted radical prostatectomy: a large European single-centre cohort with median 10-year follow-up. Eur Urol Focus 2018;4:351-9.

11. Sooriakumaran P, Haendler L, Nyberg T, Gronberg H, Nilsson A, Carlsson S, et al. Biochemical recurrence after robot-assisted radical prostatectomy in a European single-centre cohort with a minimum follow-up time of 5 years. Eur Urol 2012;62:76874.

12. Lee SH, Seo HJ, Lee NR, Son SK, Kim DK, Rha KH. Robotassisted radical prostatectomy has lower biochemical recurrence than laparoscopic radical prostatectomy: systematic review and meta-analysis. Investig Clin Urol 2017;58:152-63.

13. Abdel Raheem A, Kim DK, Santok GD, Alabdulaali I, Chung $\mathrm{BH}$, Choi YD, et al. Stratified analysis of 800 Asian patients after robot-assisted radical prostatectomy with a median 64 months of follow up. Int J Urol 2016;23:765-74.

14. Diaz M, Peabody JO, Kapoor V, Sammon J, Rogers CG, Stricker $\mathrm{H}$, et al. Oncologic outcomes at 10 years following robotic radical prostatectomy. Eur Urol 2015;67:1168-76.

15. Shao IH, Chang YH, Hou CM, Lin ZF, Wu CT. Predictors of short-term and long-term incontinence after robot-assisted radical prostatectomy. J Int Med Res 2018;46:421-9.

16. Kadono Y, Ueno S, Kadomoto S, Iwamoto H, Takezawa Y, Nakashima K, et al. Use of preoperative factors including urodynamic evaluations and nerve-sparing status for predicting urinary continence recovery after robot-assisted radical prostatectomy: nerve-sparing technique contributes to the reduction of postprostatectomy incontinence. Neurourol Urodyn 2016;35:1034-9.

17. Honda M, Kawamoto B, Morizane S, Hikita K, Muraoka K, Sejima T, et al. A prognostic model for predicting urinary incontinence after robot-assisted radical prostatectomy. Int J Med Robot 2017;13. doi: 10.1002/rcs.1780. 